VOL. $18(1978), 421-428$.

\title{
Tall profinite groups
}

\section{M.F. Hutchinson}

\begin{abstract}
A classical theorem of Camille Jordan concerning finite linear groups is used to give two structural characterisations of profinite groups which have only finitely many pairwise inequivalent, continuous, irreducible, unitary representations of each degree. Our characterisations of such groups involve properties of their open normal subgroups.
\end{abstract}

The distinction between compact groups which are tall and those which are rot has proved to be useful in the harmonic analysis of compact groups. See, for example, McMullen and Price [9] and Hutchinson [6, 7].

In this note we restrict attention to those compact groups which are totally disconnected (that is, profinite groups) and give two related structural characterisations of those profinite groups which are tall. Our proof makes use of a classical theorem of Jordan on finite linear groups.

\section{Notation}

If $G$ is a compact group, then its dual object $\hat{G}$ is a maximal set of pairwise inequivalent, continuous, irreducible, unitary representations of $G$. We say that $G$ is talz if for each positive integer $n$ there are only finitely many $\sigma \in \hat{G}$ with degree $d_{\sigma}$ equal to $n$.

If $G$ is a profinite group with neighbourhood base at the identity $\left\{N_{\alpha}\right\}_{\alpha \in A}$ consisting of open normal subgroups of $G$, then $G$ is the projective limit of the family $\left\{G / N_{\alpha}\right\}_{\alpha \in A}$ of finite groups. Moreover,

Received 7 March 1978. This work forms part of the author's doctoral dissertation done under the supervision of $\mathrm{Dr}$ J.R. McMullen. See [5] for an abstract. 
every irreducible representation $\sigma \in \hat{G}$ has kernel containing some $N_{\alpha}$, by Lemma (28.17) of Hewitt and Ross [3]. Thus we may write

$$
\hat{G}=\underset{\alpha \in A}{U}\left(G / N_{\alpha}\right)^{\wedge} \text {, }
$$

if we make the obvious identification of a representation of a quotient of $G$ with a representation of $G$. It is not surprising, therefore, that our characterisations of tall profinite groups involve properties of their open normal subgroups. Further information on profinite groups may be found in Shatz [10] and in Hewitt and Ross [2, 3].

For each positive integer $n$ let $G^{(n)}$ denote the closure of the $n$th derived subgroup of a compact group $G$.

\section{First characterisation}

LEMMA 2.1. Let $G$ be a tall compact group. Then we have:

(i) $G^{(1)}$ has finite index in $G$;

(ii) $G$ contains only finitely many open normal subgroups of index $n$ for each positive integer $n$.

Proof. (i) If $G^{(1)}$ had infinite index in $G$ then there would be infinitely many elements of $\hat{G}$ of degree 1 , contradicting the fact that $G$ is tall.

(ii) Let $N$ be an open normal subgroup of $G$ of index $n$. Since the irreducible representations of any compact group separate the points of that group (see Theorem (22.12) of Hewitt and Ross [2]) and since the square of the degree of any irreducible representation of a finite group does not exceed the order of that group, we have that $N$ contains the closed normal subgroup

$$
H=n\left\{\operatorname{ker} \sigma: \sigma \in \hat{G}, d_{\sigma}^{2} \leq n,|G: \operatorname{ker} \sigma|<\infty\right\} .
$$

Since $G$ is tall, there are only finitely many $\sigma \in \hat{G}$ with $d_{\sigma}^{2} \leq n$; so $H$ has finite index in $G$. It follows that there could only have been finitely many pairwise distinct possible choices for $N$.

LEMMA 2.2. Let $H$ be an open normal subgroup of a compact group 
$G$. Then $H$ is tali if and only if $G$ is talz.

Proof. See Lemma (3.1) of Hutchinson [6].

COROLLARY 2.3. Let $G$ be a tall compact group. Then no open normal subgroup of $G$ has an infinite abelian quotient.

Proof. This follows immediately from Lemma 2.1 (i) and Lemma 2.2.

LEMMA 2.4 (Jordan [8]). There exists a positive integer valued function $J$ defined on the positive integers such that every finite complex linear group $G$ of degree $n$ contains an abelian normal subgroup $A$ of index not exceeding $J(n)$.

We now give our first characterisation of tall profinite groups.

THEOREM 2.5. For a profinite group $G$ to be tall it is both necessary and sufficient that $G$ satisfy the following two conditions:

(i) no open normal subgroup of $G$ has an infinite abelian quotient;

(ii) $G$ contains only finitely many open normal subgroups of. index $n$ for each positive integer $n$.

Proof. The necessity of $(i)$ and $(i i)$ is contained in Corollary 2.3 and Lemma 2.1 (ii) respectively. To prove that they are sufficient, let $G$ be a profinite group satisfying $(i)$ and $(i i)$ and suppose that $G$ is not tall. Then for some positive integer $n$ we have that

$$
S=\left\{\sigma \in \hat{G}: d_{\sigma}=n\right\}
$$

is an infinite set. Now, for each $\sigma \in S, G /$ ker $\sigma$ is isomorphic to a finite complex linear group of degree $n$. Therefore, by Lemm 2.4, for each $\sigma \in S$ there is an open normal subgroup $H_{\sigma}$ of $G$ such that $H_{\sigma} \supset \operatorname{ker} \sigma, H_{\sigma} / \operatorname{ker} \sigma$ is abelian, and $\left|G: H_{\sigma}\right| \leq J(n)$. By condition (ii), we may choose an open normal subgroup $H$ of $G$ such that

$$
T=\left\{\sigma \in S: H_{\sigma}=H\right\}
$$

is an infinite set. Let $K=\bigcap_{\sigma \in T}$ ker $\sigma$. Since $H^{(1)} \subset$ ker $\sigma$ for all $\sigma \in T$, we see that $H / K$ is an infinite abelian group. But this contradicts (i); so $G$ must be tall. 
COROLLARY 2.6. Let $G$ be a profinite group which is topologically finitely generated. Then the following statements are equivalent:

(i) G is tall;

(ii) no open normal subgroup of $G$ has an infinite abelian quatient.

Proof. This follows immediately from Theorem 2.5 in view of the fact that if $G$ is topologically finitely generated, then $G$ contains only finitely many open normal subgroups of index $n$ for each positive integer $n$.

COROLLARY 2.7. Let $G$ be a prosolvable group (that is a projective limit of finite solvable groups). Then the following statements are equivalent:

(i) $G$ is tall;

(ii) no open normal subgroup of $G$ has an infinite abelian quotient;

(iii) $G^{(n)}$ has finite index in $G$ for each positive integer $n$.

\section{Second characterisation}

Our second characterisation of tall profinite groups follows immediately from Theorem 2.5 and the following lemma.

LEMMA 3.1. The following statements are equivalent for a compact group $G$ :

(i) $G$ contains infinitely many open normal subgroups of index $n$ for some positive integer $n$;

(ii) $G$ contains closed nomnal subgroups $H$ and $K$ such that $|G: H|$ is finite, $H \supset K$, and $H / K$ is isomorphic to a cartesian product of infinitely many copies of a finite simple group $S$.

Proof. We need only show that (i) implies (ii), so assume that (i) holds. Let $n$ be the smallest positive integer for which $G$ contains infinitely many open normal subgroups of index $n$. Since there are only 
finitely many groups of order $n$, there exists a finite group $A$ of order $n$ such that

$$
A=\{M \triangleleft G: G / M \cong A\}
$$

is an infinite set. Let $B$ be a minimal normal subgroup of $A$. Then $B$ is the cartesian product of a finite number of copies of a finite simple group $S$ by Satz 9.12 a) of Huppert [4]. For each $M \in A$ choose $H_{M}$ normal in $G$ such that $H_{M} \supset M$ and $H_{M} / M \cong B$. By our choice of $n$, there are only finitely many pairwise distinct such groups. Thus we may choose one of them, call it $H$, so that

$$
B=\left\{M \in A: H_{M}=H\right\}
$$

is an infinite set. Then we have that

$$
C=\{N \triangleleft H: H / N \cong S\}
$$

is an infinite set, since each $M \in B$ can be realised as the intersection of certain members of $C$. Since each $N \in C$ has no more than $|G: H|$ pairwise distinct conjugates, we may choose an infinite sequence $D=\left\{N_{i}\right\}_{i=1}^{\infty}$ of pairwise distinct members of $C$ which is closed under conjugation by elements of $G$. Then $K=\bigcap_{i=1}^{\infty} N_{i}$ is a closed normal subgroup of $G$ of infinite index in $H$. We now show that $H / K$ is isomorphic to the cartesian product of countably many copies of $S$. Choose a subsequence of $D$ by setting $Q_{1}=N_{1}$ and, if $Q_{1}, \ldots, Q_{m-1}$ have been chosen, setting $Q_{m}=N_{j}$ where $j$ is the smallest positive integer for which $N_{j} \ngtr \bigcap_{i=1}^{m-1} Q_{i}$. Clearly we have $K=\bigcap_{i=1}^{\infty} Q_{i}$. Now define a continuous homomorphism

$$
\phi: H \rightarrow \prod_{i=1}^{\infty} H / Q_{i}
$$

by setting $\phi(x)=\left(x Q_{i}\right)_{i=1}^{\infty}$ for all $x \in H$. Since $\phi$ has kernel $K$ and $H / Q_{i} \cong S$ for each $i$, our result will follow if we show that $\phi$ is surjective. For each positive integer $k$ let 


$$
q_{k}: \prod_{i=1}^{\infty} H / Q_{i} \rightarrow \prod_{i=1}^{k} H / Q_{i}
$$

be the canonical projection. Since $\phi(H)$ is closed ( $H$ is compact), it is sufficient to show that for each $k$ the composite mapping

$$
q_{k} \circ \phi: H \rightarrow \prod_{i=1}^{k} H / Q_{i}
$$

is surjective. Evidently this holds when $k=1$. Now let $k>1$ and suppose by induction that $q_{k-1} \circ \phi$ is surjective. Then, since

$$
\operatorname{ker}\left(q_{k-1} \circ \phi\right)=\bigcap_{i=1}^{k-1} Q_{i}
$$

we have that

$$
\left|H: \prod_{i=1}^{k-1} Q_{i}\right|=|S|^{k-1}
$$

Because $H / Q_{i}$ is simple, $Q_{k}$ and $\bigcap_{i=1}^{k-1} Q_{i}$ must generate $H$. Therefore, by Satz 3.12 of Huppert [4], we have

$$
\begin{aligned}
\left|H: \bigcap_{i=1}^{k} Q_{i}\right| & =\left|H: \prod_{i=1}^{k-1} Q_{i}\right| \cdot\left|\bigcap_{i=1}^{k-1} Q_{i}: \bigcap_{i=1}^{k} Q_{i}\right| \\
& =\left|H: \prod_{i=1}^{k-1} Q_{i}\right| \cdot\left|H: Q_{k}\right| \\
& =|S|^{k} .
\end{aligned}
$$

It follows that $q_{k} \circ \phi$ is surjective, as was required to be shown.

THEOREM 3.2. Let $G$ be a profinite group. Then $G$ is not tall if and only if there exists an open normal subgroup $H$ of $G$ and a closed normal subgroup $K$ of $G$ such that $K \subset H$ and either of the following conditions is satisfied:

(i) $H / K$ is an infinite abelian group;

(ii) $H / K$ is isomorphic to the cartesian product of infinitely many copies of a finite non-abelian simple group. 
REMARK. The preceding theorem says essentially that the only non tall profinite groups are the obvious ones.

\section{References}

[1] Charles W. Curtis, Irving Reiner, Representation theory of finite groups and associative algebras (Pure and Applied Mathematics, 11. Interscience [John Wiley \& Sons], New York, London, Sydney, 1962).

[2] Edwin Hewitt and Kenneth A. Ross, Abstract harmonic analysis, Volume I (Die Grundlehren der mathematischen Wissenschaften, 115. Springer-Verlag, Berlin, Göttingen, Heidelberg, 1963).

[3] Edwin Hewitt, Kenneth A. Ross, Abstract harmonic analysis, Volume II (Die Grundlehren der mathematischen Wissenschaften, 152. Springer-Verlag, Berlin, Heidelberg, New York, 1970).

[4] B. Huppert, Endliche Gruppen I (Die Grundlehren der mathematischen Wissenschaften, 134. Springer-Verlag, Berlin, Heidelberg, New York, 1967).

[5] Michael Frank Hutchinson, "Lacunary sets for connected and totally disconnected compact groups" (PhD thesis, University of Sydney, Sydney, 1977). See also: Abstract, Bull. Austral. Math. Soc. 18 (1978), 149-151.

[6] M.F. Hutchinson, "Non-tall compact groups admit infinite Sidon sets", J. Austral. Math. Soc. Ser. A 23 (1977), 467-475.

[7] M.F. Hutchinson, "Local $\Lambda$ sets for profinite groups", submitted.

[8] Camille Jordan, "Mémoire sur les équations différentielles linéaires à intégral algébrique", J. reine Angew. Math. 84 (1878), 89-215.

[9] J.F. McMullen and J.F. Price, "Rudin-Shapiro sequences for arbitrary compact groups", J. Austraz. Math. Soc. Ser. A 22 (1976), 421-430. 
[10]. Stephen S. Shatz, Profinite groups, arithmetic, and geometry (Annals of Mathematics Studies, 67. Princeton University Press, Princeton, New Jersey; University of Tokyo Press, Tokyo, 1972).

Department of Pure Mathematics, University of Sydney,

Sydney,

New South Wales. 